\title{
Novel Direct Detection Constraints on Light Dark Matter
}

\author{
Torsten Bringmann ${ }^{1}$ and Maxim Pospelov ${ }^{2,3}$ \\ ${ }^{1}$ Department of Physics, University of Oslo, Box 1048, N-0371 Oslo, Norway \\ ${ }^{2}$ Perimeter Institute for Theoretical Physics, Waterloo, Ontario N2J 2W9, Canada \\ ${ }^{3}$ Department of Physics and Astronomy, University of Victoria, Victoria, British Columbia V8P 5C2, Canada
}

(Received 5 November 2018; published 1 May 2019)

\begin{abstract}
All attempts to directly detect particle dark matter (DM) scattering on nuclei suffer from the partial or total loss of sensitivity for DM masses in the GeV range or below. We derive novel constraints from the inevitable existence of a subdominant, but highly energetic, component of DM generated through collisions with cosmic rays. Subsequent scattering inside conventional DM detectors, as well as neutrino detectors sensitive to nuclear recoils, limits the DM-nucleon scattering cross section to be below $10^{-31} \mathrm{~cm}^{2}$ for both spin-independent and spin-dependent scattering of light DM.
\end{abstract}

DOI: $10.1103 /$ PhysRevLett.122.171801

Introduction.-Attempts to discover nongravitational interactions of dark matter (DM) are a global effort, pursuing many possible avenues-perhaps as many as there are viable microscopic models that link DM with the rest of fundamental physics [1,2]. The simplicity of the early Universe suggests that DM may be realized in the form of some relic particles $[3,4]$, remnants of the big bang, which we denote here as $\chi$.

Among the very few things known about the galactic component of DM is the scale of its velocity, $v_{\chi, \text { gal }} \sim 10^{-3} c$. The energy carried by DM particles, $E_{\chi} \sim m_{\chi} v_{\chi, \text { gal }}^{2}$, can be shared with an atomic nucleus in the process of a collision and therefore, in principle, be detected [5]. The search for such DM-nucleus scatterings-commonly referred to as direct DM detection-has seen several generations of experiments with ever-improving sensitivity. In the absence of a credible positive signal, this has translated to continuously tightening limits. The latest results from the XENON1T Collaboration [6] bring the sensitivity to the cross section per nucleon below the $\sigma_{\chi}=10^{-46} \mathrm{~cm}^{2}$ level for the "optimal" DM mass range, $m_{\chi} \in 15-100 \mathrm{GeV}$. This significantly constrains many models of weak-scale DM (see, e.g., [7]).

Below that mass range, and especially below $1 \mathrm{GeV}$, the direct sensitivity to DM worsens rapidly. This is because the nuclear recoil energy becomes smaller and cannot exceed $E_{\text {recoil }}^{\max }=2 m_{\chi}^{2}\left(v_{\text {esc }}\right)^{2} / m_{A}$, where $v_{\text {esc }} \sim 540 \mathrm{~km} / \mathrm{s}$ is the galactic escape velocity and $m_{A}$ is the nuclear mass. If $E_{\text {recoil }}^{\max }$ is below some detector threshold $E_{\mathrm{thr}}$, the

Published by the American Physical Society under the terms of the Creative Commons Attribution 4.0 International license. Further distribution of this work must maintain attribution to the author(s) and the published article's title, journal citation, and DOI. Funded by SCOAP ${ }^{3}$. sensitivity completely disappears, making even cross sections parametrically larger than weak-scale cross sections (e.g., $\sigma \gg 10^{-36} \mathrm{~cm}^{2}$ ) completely undetectable.

Recently, it has been realized that several physical processes allow us to circumvent this limitation. If the scattering on the nucleus results in the emission of a photon or ejection of an atomic electron, e.g., the electromagnetic fraction of the deposited energy can be larger than for elastic nuclear recoils, improving the sensitivity for $m_{\chi}$ in the few $100 \mathrm{MeV}$ range [8-10]. Further constraints derive from multiple collisions of light DM. For example, interactions with fast moving nuclei or electrons inside the Sun can accelerate the DM above threshold for direct detection [11-13]. This contribution typically does not exceed a fraction of $\mathcal{O}\left(10^{-5}\right)$ times the total DM flux on Earth, but nevertheless greatly enhances the mass reach of existing detectors, especially for $\chi-e^{-}$scattering [12].

In this Letter, we consider another inevitable component of the DM flux, with velocities much higher than $v_{\text {esc }}$. It originates from energetic galactic cosmic rays (CRs) colliding with cold DM particles in the Milky Way halo, creating a secondary DM component of CRs with (semi-) relativistic momenta. This new component of the DM flux, called CRDM throughout this Letter, will scatter again in the detectors, but now with much greater energy available. The goal of this Letter is to make use of this idea, employing data from the most sensitive current direct detection and neutrino experiments, to establish new direct limits on DM-nucleon scattering that extend to small DM masses (formally even to $m_{\chi} \rightarrow 0$ ).

We will adopt a simple two parameter model $\left\{m_{\chi}, \sigma_{\chi}\right\}$ without reference to a specific underlying theory. For the DM-nucleon elastic cross section we assume for simplicity the isospin-singlet structure, $\sigma_{\chi n}=\sigma_{\chi p} \equiv \sigma_{\chi}$, but will consider both spin-dependent and spin-independent scattering. DM models with light $m_{\chi}$ often require 
subelectroweak-scale mediators $[14,15]$ and, therefore, can be amenable to additional constraints from cosmology, colliders, neutrino, and beam dump experiments (see, e.g., Ref. [16] for a review). However, all such constraints are necessarily model dependent, while constraints derived in this Letter have greater generality. Despite invoking DM-CR interactions, in particular, they build on the same microscopic picture of DM-nucleon scattering as adopted in the standard presentation of limits from conventional direct detection experiments.

Step 1: From CR to DM fluxes.-Compared to CR velocities, DM can be considered effectively at rest. Then, the kinetic energy transferred to a DM particle in a single collision is

$$
\begin{aligned}
T_{\chi} & =T_{\chi}^{\max } \frac{1-\cos \theta}{2}, \\
T_{\chi}^{\max } & =\frac{T_{i}^{2}+2 m_{i} T_{i}}{T_{i}+\left(m_{i}+m_{\chi}\right)^{2} /\left(2 m_{\chi}\right)},
\end{aligned}
$$

where $\theta$ is the center-of-mass system scattering angle and $T_{i} \equiv E_{i}-m_{i}$ is the kinetic energy of the incoming CR particle $i$. The (spacelike) momentum transfer in the collision is given by $Q^{2}=2 m_{\chi} T_{\chi}$. For isotropic CR-DM scattering, both $T_{\chi}$ and $Q^{2}$ thus follow a flat distribution, with $T_{\chi}$ ranging from 0 to $T_{\chi}^{\max }$. Inverting Eq. (1) gives the minimal incoming $\mathrm{CR}$ energy required to obtain a DM recoil energy $T_{\chi}$,

$$
T_{i}^{\min }=\left(\frac{T_{\chi}}{2}-m_{i}\right)\left(1 \pm \sqrt{1+\frac{2 T_{\chi}}{m_{\chi}} \frac{\left(m_{i}+m_{\chi}\right)^{2}}{\left(2 m_{i}-T_{\chi}\right)^{2}}}\right),
$$

where the $+(-)$ sign applies for $T_{\chi}>2 m_{i}\left(T_{\chi}<2 m_{i}\right)$.

The local interstellar (LIS) population of CRs is well measured and typically described by their differential intensity $d I / d R$, where $R$ is the particle's rigidity. We adopt parametrizations [17,18] for $d I_{i} / d R_{i}$ of protons and ${ }^{4} \mathrm{He}$ nuclei, the two dominant $\mathrm{CR}$ components. The differential CR flux (number of particles per area, kinetic energy, and time) is then obtained as $d \Phi / d T=$ $4 \pi(d R / d T)(d I / d R)$. For an elastic scattering cross section $\sigma_{\chi i}$, the collision rate of CR particles $i$ with energy in the range $\left[T_{i}, T_{i}+d T_{i}\right]$ inside a volume $d V$ thus becomes

$$
d \Gamma_{\mathrm{CR}_{i} \rightarrow \chi}=\sigma_{\chi i} \times \frac{\rho_{\chi}}{m_{\chi}} \frac{d \Phi_{i}^{\mathrm{LIS}}}{d T_{i}} d T_{i} d V
$$

The resulting CR-induced DM flux is thus obtained by dividing by $4 \pi d^{2}$, where $d$ is the distance to the source, implying that the volume integration reduces to an angular average over a line-of-sight (LOS) integral

$$
\frac{d \Phi_{\chi}}{d T_{i}}=\int \frac{d \Omega}{4 \pi} \int_{\operatorname{LOS}} d \ell \sigma_{\chi i} \frac{\rho_{\chi}}{m_{\chi}} \frac{d \Phi_{i}}{d T_{i}} \equiv \sigma_{\chi i} \frac{\rho_{\chi}^{\text {local }}}{m_{\chi}} \frac{d \Phi_{i}^{\mathrm{LIS}}}{d T_{i}} D_{\text {eff }} .
$$

Here we introduced an effective distance $D_{\text {eff }}$ out to which we take into account CRs as the source of a possible highvelocity tail in the DM velocity distribution. The local gradient in the DM density is relatively well constrained $[19,20]$, and that in the cosmic-ray density is very small [21]. The main uncertainty in $D_{\text {eff }}$ thus derives from the extension of the diffusion zone, which reaches out to at least a few kiloparsec from the galactic disk [22-24]. Assuming a Navarro-Frenk-White profile $[25,26]$ for the DM distribution and a homogeneous CR distribution, e.g., performing the full line-of-sight integration out to $1 \mathrm{kpc}(10 \mathrm{kpc})$ results in $D_{\text {eff }}=0.997 \mathrm{kpc}\left(D_{\text {eff }}=8.02 \mathrm{kpc}\right)$. Using Eq. (1), we can finally express the DM flux in terms of the DM energy by integrating over all CR energies $T_{i}$,

$$
\frac{d \Phi_{\chi}}{d T_{\chi}}=\int_{0}^{\infty} d T_{i} \frac{d \Phi_{\chi}}{d T_{i}} \frac{1}{T_{\chi}^{\max }\left(T_{i}\right)} \Theta\left[T_{\chi}^{\max }\left(T_{i}\right)-T_{\chi}\right] .
$$

The flat distribution over recoil energies that follows from Eq. (1) for isotropic scattering is an assumption that we modify by the inclusion of the hadronic elastic scattering form factor in the simplest dipole form [27],

$$
G_{i}\left(Q^{2}\right)=1 /\left(1+Q^{2} / \Lambda_{i}^{2}\right)^{2} .
$$

Here, $\Lambda_{i}$ scales inversely proportional with the charge radius and is hence smaller for heavier nuclei; for proton (helium) scattering due to a vector current, one has $\Lambda_{p} \simeq$ $770 \mathrm{MeV}\left(\Lambda_{\mathrm{He}} \simeq 410 \mathrm{MeV}\right)$ [28]). We thus relate the scattering cross section to that in the pointlike limit by

$$
\frac{d \sigma_{\chi i}}{d \Omega}=\left.\frac{d \sigma_{\chi i}}{d \Omega}\right|_{Q^{2}=0} G_{i}^{2}\left(2 m_{\chi} T_{\chi}\right) .
$$

Putting everything together, we expect the following CR-induced DM flux:

$$
\begin{aligned}
\frac{d \Phi_{\chi}}{d T_{\chi}}= & D_{\mathrm{eff}} \frac{\rho_{\chi}^{\text {local }}}{m_{\chi}} \\
& \times \sum_{i} \sigma_{\chi i}^{0} G_{i}^{2}\left(2 m_{\chi} T_{\chi}\right) \int_{T_{i}^{\min }}^{\infty} d T_{i} \frac{d \Phi_{i}^{\mathrm{LIS}} / d T_{i}}{T_{\chi}^{\max }\left(T_{i}\right)} .
\end{aligned}
$$

Here, we only include $i \in\left\{p,{ }^{4} \mathrm{He}\right\}$ in the sum. In Fig. 1, we plot these CRDM fluxes for various DM masses, for spin-independent $\sigma_{\chi}=\sigma_{n}=\sigma_{p}$. The contribution from helium can be even larger than that from protons, but is form-factor-suppressed at large recoil energies. The flux is related to the 1D velocity distribution $f(v)$, more familiar in the context of direct DM searches, as $f(v)=$ $m_{\chi}^{2}\left(\rho_{\chi}^{\text {local }}\right)^{-1} \gamma^{3} d \Phi_{\chi} / d T_{\chi}$. For illustration, we compare this to the Maxwellian distribution of the standard halo model 


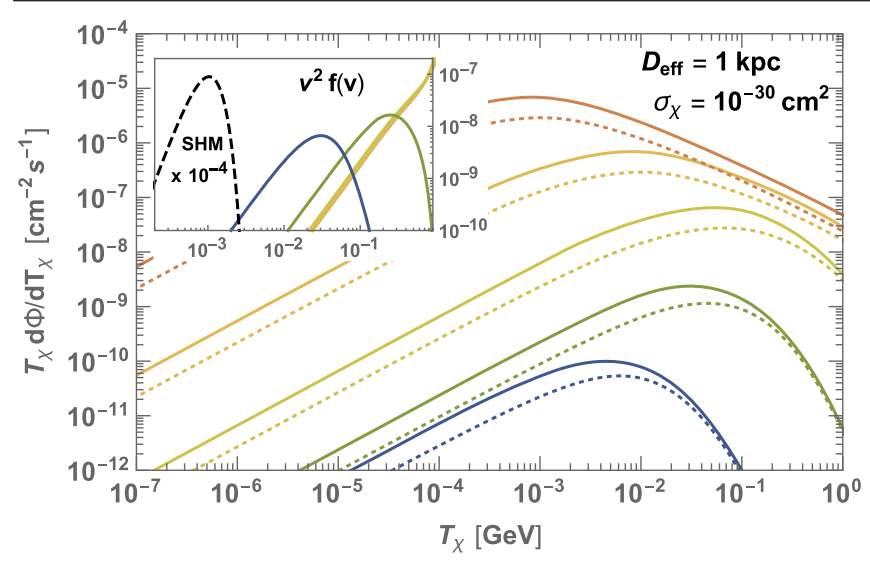

FIG. 1. Expected flux of CRDM for different DM masses $m_{\chi}=0.001,0.01,0.1,1,10 \mathrm{GeV}$ (from top to bottom). Dotted lines show the contribution from $\mathrm{CR}$ proton scattering alone. The flux is directly proportional to the effective distance $D_{\text {eff }}$ and the elastic scattering cross section $\sigma_{\chi}$, chosen here as indicated. (Inset) Compares the corresponding 1D velocity distributions $f(v)$, in units where $c=1$, to that of the standard halo model (SHM) (dashed line).

[29], displayed as a dashed line in the inset. As expected, the CRDM population peaks at (semi-)relativistic velocities and is highly subdominant at the galactic DM velocities typically considered.

Step 2: Attenuation of CRDM flux.-Very large scattering cross sections generally constitute a blind spot for direct DM detection, because they would lead to a significant attenuation of the DM flux from the top of the atmosphere to the location of the detector [30-33]. The degradation in energy should also occur for the CRDM component, and we can estimate the energy loss of DM particles propagating through a medium as

$$
\frac{d T_{\mathrm{DM}}}{d x}=-\sum_{N} n_{N} \int_{0}^{T_{r}^{\max }} \frac{d \sigma_{\chi N}}{d T_{r}} T_{r} d T_{r}
$$

Here, $T_{r}$ refers to the energy lost by a CRDM particle in a collision with nucleus $N$. This process, in analogy with neutrino scattering, can be elastic, quasielastic, or inelastic. The latter two are likely to dominate at high energies $T_{\chi}>$ few $100 \mathrm{MeV}$. (In a quasielastic process, one or more nucleons are dislodged from $N$, while in an inelastic process, additional hadrons are created in the $\chi-N$ collision.) In this Letter, we will limit ourselves to elastic scattering, leaving a more elaborate treatment for future considerations. Using the uniform distribution of the nuclear recoil energy for isotropic scattering, we have $d \sigma_{N} / d T_{r}=\sigma_{N} / T_{r}^{\max }$, and hence

$$
\begin{aligned}
\frac{d T_{\chi}}{d x} & =-\frac{1}{2} \sum_{N} n_{N} \sigma_{\chi N} T_{r}^{\max } \approx-\frac{1}{2 m_{\chi} \ell}\left(T_{\chi}^{2}+2 m_{\chi} T_{\chi}\right), \\
\text { where } \ell^{-1} & \equiv \sum_{N} n_{N} \sigma_{\chi N} \frac{2 m_{N} m_{\chi}}{\left(m_{N}+m_{\chi}\right)^{2}} .
\end{aligned}
$$

In the last step, we have assumed $T_{\chi} \ll m_{N}$ in Eq. (1). Integrating this equation, we can relate, very approximately, the differential DM flux at depth $z$ to the one at the top of the atmosphere as

$$
\frac{d \Phi_{\chi}}{d T_{\chi}^{z}}=\left(\frac{d T_{\chi}}{d T_{\chi}^{z}}\right) \frac{d \Phi_{\chi}}{d T_{\chi}}=\frac{4 m_{\chi}^{2} e^{z / \ell}}{\left(2 m_{\chi}+T_{\chi}^{z}-T_{\chi}^{z} e^{z / \ell}\right)^{2}} \frac{d \Phi_{\chi}}{d T_{\chi}},
$$

where $d \Phi_{\chi} / d T_{\chi}$ needs to be evaluated at

$$
T_{\chi}=T_{\chi}^{0}\left(T_{\chi}^{z}\right)=2 m_{\chi} T_{\chi}^{z} e^{z / \ell}\left(2 m_{\chi}+T_{\chi}^{z}-T_{\chi}^{z} e^{z / \ell}\right)^{-1} .
$$

For $T_{\chi}^{0} \ll m_{\chi}$, our treatment of the energy attenuation reduces to that previously considered in Ref. [33].

For the mean free path of the DM particles $\ell$, we use DarkSUSY [34] to calculate the average density $n_{N}$ of Earth's 11 most abundant elements between surface and depth $z$ (based on mass density profiles from Ref. [35]). We also need to relate the nuclear cross sections to the one on nucleons, $\sigma_{\chi}$. For spin-independent scattering, there is the usual coherent enhancement, leading to

$$
\sigma_{\chi N}=\sigma_{\chi}^{\mathrm{SI}} A^{2}\left(\frac{m_{N}\left(m_{\chi}+m_{p}\right)}{m_{p}\left(m_{\chi}+m_{N}\right)}\right)^{2} .
$$

We neglect nuclear form factors in obtaining $\ell$. Along with the energy-loss ansatz (9), as compared to full numerical simulations [33], this leads to conservative limits.

Step 3: CRDM scattering in detectors.-Once a CRDM particle reaches a detector at depth $z$, it can transfer (part of its) energy to a target nucleus inside the detector. Exploiting completely analogous formulas to the case of DM $\rightarrow \mathrm{CR}$ scattering discussed above, in particular, the flat distribution of the target nucleus recoil energy $T_{N}$ for a given DM energy, we find the differential recoil rate per target nucleus to be

$$
\frac{d \Gamma_{N}}{d T_{N}}=\sigma_{\chi N}^{0} G_{N}^{2}\left(2 m_{N} T_{N}\right) \int_{T_{\chi}\left(T_{\chi}^{z, \min }\right)}^{\infty} \frac{d T_{\chi}}{T_{r, N}^{\max }\left(T_{\chi}^{z}\right)} \frac{d \Phi_{\chi}}{d T_{\chi}} .
$$

Here $G_{N}\left(Q^{2}\right)$ is a nuclear form factor and $d \Phi_{\chi} / d T_{\chi}$ is given in Eq. (8); the quantities $T_{r, N}^{\max }$ and $T_{\chi}^{z, \min }$ follow from Eqs. (1) and (2), by replacing $\chi \rightarrow N$ and $i \rightarrow \chi$.

The broad energy distribution of CRDM particles allows us, based on Eq. (14), to use both conventional direct detection and neutrino experiments to set novel limits on $\sigma_{\chi}$. It is clear that for small enough $\sigma_{\chi}$ the overburden mass above the detectors is transparent to CRDM, and the overall strength of the signal hence scales as $\sigma_{\chi}^{2}$. For large cross sections, on the other hand, the strong attenuation of the CRDM energy as given in Eq. (12) also leads to an exponential suppression of the signal.

Resulting limits.-We begin by addressing constraints from conventional direct detection experiments, which we derive from reported limiting values for heavy DM cross 
sections on nucleons as a function of the DM particle mass, $\sigma_{\mathrm{DM}}^{\mathrm{SI}, \lim }\left(m_{\mathrm{DM}}\right)$. Assuming a nonrelativistic DM velocity distribution $f_{\mathrm{NR}}(v)$, and hence a standard DM flux of $d \Phi_{\mathrm{DM}} / d T_{\mathrm{DM}}=m_{\mathrm{DM}}^{-2} \rho_{\mathrm{DM}}^{\text {local }} f_{\mathrm{NR}}$, we can relate the count rate per target nucleus $N$ to the average heavy DM-nucleus cross section $\sigma_{\gamma N}^{\mathrm{DM}}$ inside the experimentally accessible window of recoil energies $T_{N} \in\left\{T_{1}, T_{2}\right\}$. In the limit of large DM masses, this gives

$$
\begin{aligned}
\Gamma_{N}^{\mathrm{DM}} & =\int_{T_{1}}^{T_{2}} d T_{N} \sigma_{\chi N}^{\mathrm{DM}} \int_{0}^{\infty} d T_{\mathrm{DM}} \frac{d \Phi_{\mathrm{DM}}}{d T_{\mathrm{DM}}} \frac{\Theta\left[T_{N}^{\max }\left(T_{\mathrm{DM}}\right)-T_{N}\right]}{T_{N}^{\max }\left(T_{\mathrm{DM}}\right)} \\
& \simeq \kappa \frac{\sigma_{\chi N}^{\mathrm{DM}}}{m_{\mathrm{DM}}}\left(\bar{v} \rho_{\mathrm{DM}}\right)^{\text {local }} \text { for } m_{\mathrm{DM}} \gg m_{N} .
\end{aligned}
$$

Here $\bar{v}$ denotes the mean DM velocity and $\kappa$ is an $\mathcal{O}(1)$ constant that, for a Maxwellian distribution, equals $\kappa=\exp \left[-2 T_{1} /\left(\pi m_{N} \bar{v}^{2}\right)\right]-\exp \left[-2 T_{2} /\left(\pi m_{N} \bar{v}^{2}\right)\right]$.

In order to constrain the CRDM component, we now need to compare Eq. (15) with Eq. (14), taking into account that $\sigma_{\chi N}^{0}$ is evaluated for $m_{\mathrm{DM}} \gg m_{N}$ only in the former case. For spin-independent scattering, we can use Eq. (13) to compute the ratio of these cross sections. Realizing that the coherence factors for $\sigma_{\chi N}$ are identical between ordinary DM and CRDM scattering then allows us to recast conventional limits on the scattering rate $\sigma_{\mathrm{DM}}^{\mathrm{SI}, \mathrm{lim}}$ per nucleon to an equivalent limit resulting from the CRDM component

$$
\begin{aligned}
\sigma_{\chi}^{\mathrm{SI}, \mathrm{lim}}= & \kappa\left(\bar{v} \rho_{\mathrm{DM}}\right)^{\operatorname{local}}\left(\frac{m_{\chi}+m_{N}}{m_{\chi}+m_{p}}\right)^{2}\left(\frac{\sigma_{\mathrm{DM}}^{\mathrm{SI}, \mathrm{lim}}}{m_{\mathrm{DM}}}\right)_{m_{\mathrm{DM}} \rightarrow \infty} \\
& \times\left(\int_{T_{1}}^{T_{2}} d T_{N} \int_{T_{\chi}\left(T_{\chi}^{z, \text { min }}\right)}^{\infty} \frac{d T_{\chi}}{T_{r, N}^{\max }} \frac{d \Phi_{\chi}}{d T_{\chi}}\right)^{-1} .
\end{aligned}
$$

For the recent Xenon 1T data ([6], Fig. 5), e.g., one has $\sigma_{\mathrm{DM}}^{\mathrm{SI}, \mathrm{lim}} / m_{\mathrm{WIMP}}=8.3 \times 10^{-49} \mathrm{~cm}^{2} / \mathrm{GeV}$ for $m_{\chi} \gtrsim 100 \mathrm{GeV}$, and $T_{\mathrm{Xe}} \in[4.9,40.9] \mathrm{keV}$ implies $\kappa \simeq 0.23$. The resulting limits on $\sigma_{\chi}$ are shown in Fig. 2 for different assumptions about the size of the diffusion zone (with solid lines corresponding to an ultraconservative choice). For small DM masses, these limits exclude cross sections in the range $10^{-31} \lesssim \sigma_{\chi}^{\mathrm{SI}} \lesssim 10^{-28} \mathrm{~cm}^{2}$, almost independently of $m_{\chi}$. Clearly, these constraints are highly complementary to existing limits on light DM [33,36-41]. Direct detection of light energetic dark sector particles was also discussed in Ref. [42].

Due to its shallow location, MiniBooNE [46] gives a particular advantage in limiting CRDM fluxes with large scattering cross sections that prevent $\chi$ from reaching deeply placed experiments. We utilize the measurement of elastic $\nu-p$ scattering [47] and a recent DM run [48] that allows us to extract the beam-unrelated scattering rate. Requiring the scattering rate of CRDM on protons at MiniBooNE depth not to exceed the beam-unrelated background, we obtain (see Supplemental Material [49])

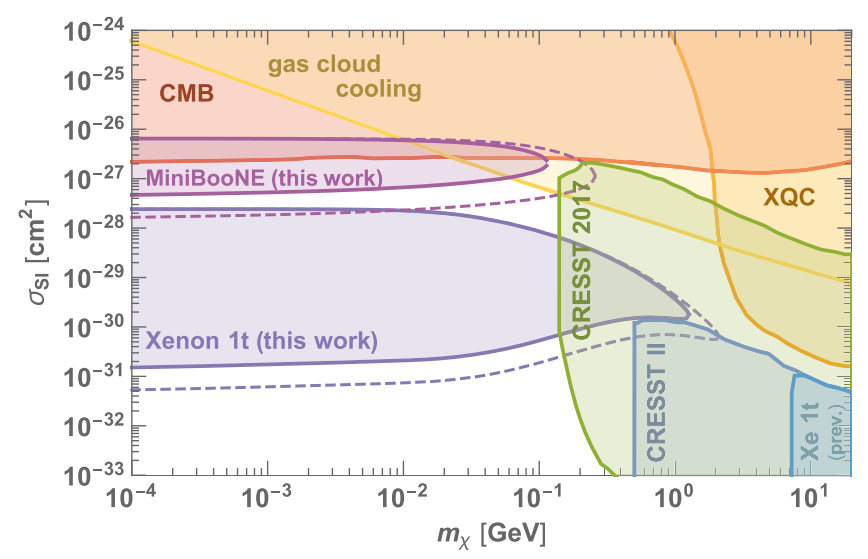

FIG. 2. Constraints on spin-independent DM-nucleon scattering imposed by the XENON-1T and MiniBooNE experiments. Solid (dashed) lines assume a CR density that equals, on average, the local value out to a distance of $1 \mathrm{kpc}(10 \mathrm{kpc})$. We compare our limits to those deriving from cosmic microwave background (CMB) observations [40], gas cloud cooling [38], the x-ray quantum calorimeter experiment (XQC) [39], and a selection of direct detection experiments [43-45] after taking into account the absorption of DM in soil and atmosphere [33].

$$
\Gamma_{p}\left(T_{p}>35 \mathrm{MeV}\right)<1.5 \times 10^{-32} \mathrm{~s}^{-1} .
$$

This additional exclusion region is also shown in Fig. 2.

Strong constraints on spin-dependent scattering, finally, can be obtained from proton upscattering by CRDM in neutrino detectors like Borexino [52]. From a search for events with higher energy than solar neutrino scattering $[53,54]$, we deduce that the limiting scattering rate per proton is (see Supplemental Material [49])

$$
\Gamma_{p}\left(T_{e}>12.5 \mathrm{MeV}\right)<2 \times 10^{-39} \mathrm{~s}^{-1} .
$$

To apply this limit, we need to convert the proton recoil energy to an apparent electron $T_{e}$ equivalent. For liquid scintillators the recoil energy of the nucleus $T_{N}$ and the detected energy $T_{e}$ are related by the empirical law

$$
T_{e}\left(T_{N}\right)=\int_{0}^{T_{N}} \frac{d T_{N}}{1+k_{B}\left\langle d T_{N} / d x\right\rangle},
$$

where $k_{B}$ is a material-dependent constant. Following the procedure outlined in Ref. [55], and thus using PSTAR tables from [56] for $\left\langle d T_{N} / d x\right\rangle$, we numerically tabulate and invert Eq. (19) for pseudocumene (the scintillator used by Borexino). The resulting constraint on spin-dependent scattering is plotted in Fig. 3. Here the CRDM component is produced exclusively by $p-\chi$ collisions, since ${ }^{4} \mathrm{He}$ nuclei do not carry spin. For the mean free path in Eq. (10), we assumed exclusively elastic scattering on nuclei as derived from spin-dependent couplings $\sigma_{\chi}=\sigma_{n}=\sigma_{p}$ to nucleons (dashed and solid lines). In reality, quasielastic scattering on nucleons would dominate for energy transfers 


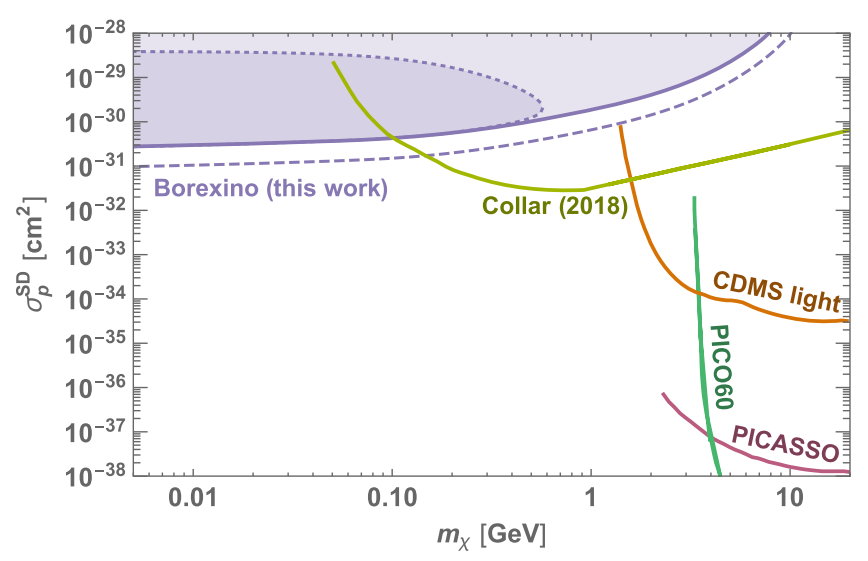

FIG. 3. Constraints on the spin-dependent part of the cross section imposed by Borexino. Solid and dashed lines as in Fig. 2. Dotted lines result from adopting the much greater stopping power expected for spin-independent scattering (so this contour also applies to $\sigma_{\mathrm{SI}}$ ). For comparison, we indicate limits from the direct detection experiments CDMS light [57], PICO60 [58], and PICASSO [59], as well as from delayed-coincidence searches in near-surface detectors by Collar [41].

above typical nuclear binding energies. While a full treatment of these processes is beyond the scope of this Letter, we indicate for comparison (dotted lines) the limits that would result in the extreme case of adopting a stopping power as efficient as in the case of spin-independent scattering [cf. Eq. (13)]. For $m_{\chi} \lesssim 0.5 \mathrm{GeV}$ we thus find highly competitive limits on (both spin-independent and) spin-dependent scattering with protons, independent of the attenuation of the CRDM flux.

Conclusions.-We have shown that the DM-nucleon interaction cross section $\sigma_{\chi}$ necessarily generates a small but very energetic component of the DM flux, the CRDM. Subsequent scattering of CRDM in DM and neutrino detectors leads to novel limits on $\sigma_{\chi}$ in the $\left\{m_{\chi}, \sigma_{\chi}\right\}$ domains that previously were thought to be completely inaccessible for direct detection. Our results thus complement and strengthen previous studies addressing the alteration of the $\mathrm{CR}$ spectrum, the generation of gamma rays in the collision of CR with DM, as well as cosmological constraints on $\chi$-nucleon interactions [32,37,40,60,61]. All routines to calculate these new limits have been implemented in DarkSUSY [34] and publicly released with version 6.2 of this code [62]. While our limits are generic and derived with a minimum set of assumptions, further refinements of the limits can be achieved within specific models, where $\sigma_{\chi}$ and its energy dependence are expressed in terms of couplings and masses of an underlying microscopic model.

We thank John Beacom, Christopher Cappiello, Juan Collar, Felix Kling, and Shafi Mahdawi for useful discussions and input on a previous version of the manuscript. T. B. wishes to warmly thank Perimeter Institute for
Theoretical Physics for generous support and hospitality during the preparation of this manuscript. Research at Perimeter Institute is supported by the Government of Canada through the Department of Innovation, Science, and Economic Development and by the Province of Ontario through the Ministry of Research, Innovation, and Science.

[1] G. Bertone, D. Hooper, and J. Silk, Phys. Rep. 405, 279 (2005).

[2] J. L. Feng, Annu. Rev. Astron. Astrophys. 48, 495 (2010).

[3] B. W. Lee and S. Weinberg, Phys. Rev. Lett. 39, 165 (1977).

[4] P. Gondolo and G. Gelmini, Nucl. Phys. B360, 145 (1991).

[5] M. W. Goodman and E. Witten, Phys. Rev. D 31, 3059 (1985).

[6] E. Aprile et al. (XENON Collaboration), Phys. Rev. Lett. 121, 111302 (2018).

[7] P. Athron et al. (GAMBIT Collaboration), Eur. Phys. J. C 77, 879 (2017).

[8] C. Kouvaris and J. Pradler, Phys. Rev. Lett. 118, 031803 (2017).

[9] M. Ibe, W. Nakano, Y. Shoji, and K. Suzuki, J. High Energy Phys. 03 (2018) 194.

[10] M. J. Dolan, F. Kahlhoefer, and C. McCabe, Phys. Rev. Lett. 121, 101801 (2018).

[11] C. Kouvaris, Phys. Rev. D 92, 075001 (2015).

[12] H. An, M. Pospelov, J. Pradler, and A. Ritz, Phys. Rev. Lett. 120, 141801 (2018).

[13] T. Emken, C. Kouvaris, and N. G. Nielsen, Phys. Rev. D 97, 063007 (2018).

[14] C. Boehm and P. Fayet, Nucl. Phys. B683, 219 (2004).

[15] M. Pospelov, A. Ritz, and M. B. Voloshin, Phys. Lett. B 662, 53 (2008).

[16] M. Battaglieri et al. (BDX Collaboration), arXiv:1607.01390.

[17] S. Della Torre et al., in Proceedings of the 25th European Cosmic Ray Symposium (ECRS 2016) Turin, Italy, 2016 (2016).

[18] M. J. Boschini et al., Astrophys. J. 840, 115 (2017).

[19] R. Catena and P. Ullio, J. Cosmol. Astropart. Phys. 08 (2010) 004.

[20] M. Benito, A. Cuoco, and F. Iocco, J. Cosmol. Astropart. Phys. 03 (2019) 033.

[21] C. Evoli, D. Gaggero, D. Grasso, and L. Maccione, Phys. Rev. Lett. 108, 211102 (2012).

[22] C. Evoli, D. Gaggero, D. Grasso, and L. Maccione, J. Cosmol. Astropart. Phys. 10 (2008) 018; 04 (2016) E01.

[23] I. V. Moskalenko, S. G. Mashnik, and A. W. Strong, in Proceedings of the 27th International Cosmic Ray Conference (ICRC 2001) Hamburg, Germany, 2001 (2001), p. 1836.

[24] T. Bringmann, F. Donato, and R. A. Lineros, J. Cosmol. Astropart. Phys. 01 (2012) 049.

[25] J. F. Navarro, C. S. Frenk, and S. D. M. White, Astrophys. J. 462, 563 (1996).

[26] M. Ackermann et al. (Fermi-LAT Collaboration), Astrophys. J. 761, 91 (2012).

[27] C. F. Perdrisat, V. Punjabi, and M. Vanderhaeghen, Prog. Part. Nucl. Phys. 59, 694 (2007).

[28] I. Angeli, At. Data Nucl. Data Tables 87, 185 (2004). 
[29] A. K. Drukier, K. Freese, and D. N. Spergel, Phys. Rev. D 33, 3495 (1986).

[30] G. D. Starkman, A. Gould, R. Esmailzadeh, and S. Dimopoulos, Phys. Rev. D 41, 3594 (1990).

[31] G. D. Mack, J. F. Beacom, and G. Bertone, Phys. Rev. D 76, 043523 (2007).

[32] D. Hooper and S. D. McDermott, Phys. Rev. D 97, 115006 (2018).

[33] T. Emken and C. Kouvaris, Phys. Rev. D 97, 115047 (2018).

[34] T. Bringmann, J. Edsjö, P. Gondolo, P. Ullio, and L. Bergström, J. Cosmol. Astropart. Phys. 07 (2018) 033.

[35] W. F. McDonough, Treatise Geochem. 2, 568 (2003).

[36] A. L. Erickcek, P. J. Steinhardt, D. McCammon, and P. C. McGuire, Phys. Rev. D 76, 042007 (2007).

[37] V. Gluscevic and K. K. Boddy, Phys. Rev. Lett. 121, 081301 (2018).

[38] A. Bhoonah, J. Bramante, F. Elahi, and S. Schon, Phys. Rev. Lett. 121, 131101 (2018).

[39] M. S. Mahdawi and G. R. Farrar, J. Cosmol. Astropart. Phys. 10 (2018) 007.

[40] W. L. Xu, C. Dvorkin, and A. Chael, Phys. Rev. D 97, 103530 (2018).

[41] J. I. Collar, Phys. Rev. D 98, 023005 (2018).

[42] Y. Cui, M. Pospelov, and J. Pradler, Phys. Rev. D 97, 103004 (2018).

[43] G. Angloher et al. (CRESST Collaboration), Eur. Phys. J. C 76, 25 (2016).

[44] G. Angloher et al. (CRESST Collaboration), Eur. Phys. J. C 77, 637 (2017).

[45] E. Aprile et al. (XENON Collaboration), Phys. Rev. Lett. 119, 181301 (2017).

[46] G. Karagiorgi, A. Aguilar-Arevalo, J. M. Conrad, M. H. Shaevitz, K. Whisnant, M. Sorel, and V. Barger, Phys. Rev. D 75, 013011 (2007); 80, 099902(E) (2009).
[47] A. A. Aguilar-Arevalo et al. (MiniBooNE Collaboration), Phys. Rev. D 91, 012004 (2015).

[48] A. A. Aguilar-Arevalo et al. (MiniBooNE Collaboration), Phys. Rev. Lett. 118, 221803 (2017).

[49] See Supplemental Material at http://link.aps.org/ supplemental/10.1103/PhysRevLett.122.171801 for details about the use of MiniBooNE and Borexino data to infer the limiting counting rate per proton, Eqs. (17) and (18) in the main text, which additionally includes Refs. [50,51].

[50] G. Bellini et al. (Borexino Collaboration), Phys. Rev. D 82, 033006 (2010).

[51] M. Agostini et al. (Borexino Collaboration), arXiv: 1709.00756.

[52] G. Alimonti et al. (Borexino Collaboration), Astropart. Phys. 16, 205 (2002).

[53] G. Bellini et al. (Borexino Collaboration), Phys. Rev. C 81, 034317 (2010).

[54] G. Bellini et al. (Borexino Collaboration), Phys. Rev. D 88, 072010 (2013).

[55] B. Dasgupta and J. F. Beacom, Phys. Rev. D 83, 113006 (2011).

[56] http://physics.nist.gov.

[57] R. Agnese et al. (SuperCDMS Collaboration), Phys. Rev. D 97, 022002 (2018).

[58] C. Amole et al. (PICO Collaboration), Phys. Rev. Lett. 118, 251301 (2017).

[59] E. Behnke et al., Astropart. Phys. 90, 85 (2017).

[60] R. H. Cyburt, B. D. Fields, V. Pavlidou, and B. D. Wandelt, Phys. Rev. D 65, 123503 (2002).

[61] C. V. Cappiello, K. C. Y. Ng, and J. F. Beacom, Phys. Rev. D 99, 063004 (2019).

[62] https://darksusy.hepforge.org. 